\title{
Demonstration of the steady-state fluctuation theorem from a single trajectory
}

\author{
G M Wang, D M Carberry, J C Reid, E M Sevick and D J Evans \\ Research School of Chemistry, The Australian National University, Canberra 0200, ACT, \\ Australia \\ Received 5 October 2005 \\ Published 28 October 2005 \\ Online at stacks.iop.org/JPhysCM/17/S3239
}

\begin{abstract}
The fluctuation theorem (FT) quantifies the probability of Second Law of Thermodynamics violations in small systems over short timescales. While this theorem has been experimentally demonstrated for systems that are perturbed from an initial equilibrium state, there are a number of studies suggesting that the theorem applies asymptotically in the long time limit to systems in a nonequilibrium steady state. The asymptotic application of the FT to such nonequilibrium steady-states has been referred to in the literature as the steady-state fluctuation theorem (or SSFT). In 2005 Wang et al demonstrated experimentally an integrated form of the SSFT using a colloidal bead that was weakly held in a circularly translating optical trap. Moreover, they showed that the integrated form of the FT may, for certain systems, hold under non-equilibrium steady states for all time, and not just in the long time limit, as suggested by the SSFT. While demonstration of the integrated forms of these theorems is compact and illustrative, a proper demonstration shows the theorem directly, rather than in its integrated form. In this paper, we present experimental results that demonstrate the SSFT directly, and show that the FT can hold for all time under nonequilibrium steady states.
\end{abstract}

\section{Introduction}

The fluctuation theorem (FT) of Evans et al [1,2] describes how a system's irreversibility develops in time from a completely time-reversible system at short observation times, to a thermodynamically irreversible one at infinitely long times. That is, it bridges the microscopic and macroscopic descriptions, relating a system's time-reversible equations of motion to the Second Law. Specifically, the FT relates the relative probabilities of observing trajectories of duration $t$ and their conjugate anti-trajectories, each characterized by the dissipation function, $\Omega_{t}$, taking on arbitrary values $A$ and $-A$, respectively:

$$
\frac{P\left(\Omega_{t}=-A\right)}{P\left(\Omega_{t}=A\right)}=\exp (-A)
$$


The dissipation function, $\Omega_{t}$, is a dimensionless dissipated energy that is accumulated along the system's trajectory; expressions for $\Omega_{t}$ differ from system to system. In general, it is an extensive property, i.e., its magnitude scales with system size and observation time, $t$. Thus, equation (1) also shows that as the system size gets larger or the observation time gets longer, anti-trajectories become rare and it becomes overwhelmingly likely that the system appears time-irreversible, in accord with the Second Law. This relationship between the FT and the Second Law is more clearly evident in the integrated form of the FT:

$$
\frac{P\left(\Omega_{t}<0\right)}{P\left(\Omega_{t}>0\right)}=\left\langle\exp \left(-\Omega_{t}\right)\right\rangle_{\Omega_{t}>0}
$$

In addition, equation (1) shows that the ensemble average of the dissipation function is positive for all $t$ and for any system size; i.e., $\left\langle\Omega_{t}\right\rangle \geqslant 0$ which is referred to as the Second Law inequality [3].

In the literature, the reader will find two different labels for the fluctuation theorem, depending upon how the theorem is applied. The transient fluctuation theorem or TFT is simply equation (1) applied to transient systems, i.e., systems that evolve from a known initial equilibrium state towards a final equilibrium or non-equilibrium steady-state. The steady-state fluctuation theorem or SSFT refers to the steady-state application of the theorem, where the dissipation function is evaluated over trajectory segments of duration $t$, sampled wholly under non-equilibrium steady-state conditions. When $\Omega_{t}$ is evaluated for deterministic, steady-state trajectories, the theorem holds asymptotically,

$$
\lim _{t \rightarrow \infty} \frac{P\left(\Omega_{t}=-A\right)}{P\left(\Omega_{t}=A\right)}=\exp (-A) \quad \text { for all } A,
$$

which is equivalent to the form of the SSFT given in the literature. The integrated form of the SSFT is

$$
\lim _{t \rightarrow \infty} \frac{P\left(\Omega_{t}<0\right)}{P\left(\Omega_{t}>0\right)}=\left\langle\exp \left(-\Omega_{t}\right)\right\rangle_{\Omega_{t}>0}
$$

As we show in this paper, the asymptotic limit in the SSFT is a result of approximations made in the argument of the theorem, $\Omega_{t}$. When we are able to express $\Omega_{t}$ exactly, the asymptotic limit is no longer needed and the operative theorem under steady-state conditions is the FT, equation (1).

To experimentally demonstrate the FT under steady-state conditions, we chose a system where the dissipation function can be approximated for deterministic dynamics and expressed exactly for stochastic or Langevin dynamics. This system is based upon the drag experiment used by Wang et al $[4,5]$ where a colloidal particle is weakly held in a stationary optical trap that is translated uniformly with velocity, $\mathbf{v}_{\text {opt }}$, starting at $t=0$. Initially the particle's position in the harmonic well is distributed according to an equilibrium Boltzmann distribution with an average particle velocity of 0 . With trap translation, the particle is displaced from its equilibrium position until, at some later time, the average velocity of the particle is equal to the trap velocity and the average particle position is determined by a balance between the optical force and hydrodynamic drag. From this point, the system is in a non-equilibrium steady state. In their original experiment, Wang and colleagues evaluated the dissipation function, constructed using deterministic dynamics from an equilibrium initial condition and thereby demonstrated an integrated form of the FT, equation (2), under transient conditions. More recently, Wang et al [5] demonstrated the integrated forms of the FT and the SSFT for steady-state translation of a particle-filled optical trap.

In this paper, we demonstrate the FT directly, rather than indirectly through the integrated form of the theorems. We do this by evaluating the dissipation function over a single trajectory 
of an optically trapped colloidal particle, held in a circularly translating trap. Consistent with previous theoretical predictions in the literature, we demonstrate experimentally that the FT holds asymptotically in the long time limit. However the asymptotic limit is only necessary when $\Omega_{t}$ is derived approximately. When the dissipation function, $\Omega_{t}$, is derived exactly for steady-state conditions, the FT holds for all time, including short times.

\subsection{Deterministic derivation of an approximate dissipation function for steady-state trajectories}

For a Newtonian, deterministic system, a system's state is described in terms of the coordinates $\mathbf{q}$ and momenta $\mathbf{p}$ of all constituent molecules, including solvent molecules, and is represented by a point in phase space, $\Gamma \equiv\{\mathbf{q}, \mathbf{p}\}$. For every trajectory that is initiated at $\boldsymbol{\Gamma}_{0} \equiv\left\{\mathbf{q}_{0}, \mathbf{p}_{0}\right\}$ and terminates at $\boldsymbol{\Gamma}_{t} \equiv\left\{\mathbf{q}_{t}, \mathbf{p}_{t}\right\}$ in a system of reversible dynamics, there is a unique conjugate or anti-trajectory that starts at $\boldsymbol{\Gamma}_{0}^{*} \equiv\left\{\mathbf{q}_{t},-\mathbf{p}_{t}\right\}$ and ends at $\boldsymbol{\Gamma}_{t}^{*} \equiv\left\{\mathbf{q}_{0},-\mathbf{p}_{0}\right\}$. Let $\delta V\left(\boldsymbol{\Gamma}_{s} \equiv\left\{\mathbf{q}_{s}, \mathbf{p}_{s}\right\}\right)$ represent a volume element of a bundle of trajectories at time $s$. Then the corresponding bundle of conjugate trajectories or anti-trajectories has the volume $\delta V\left(\boldsymbol{\Gamma}_{s}^{*} \equiv\left\{\mathbf{q}_{t-s},-\mathbf{p}_{t-s}\right\}\right)$ at time $s$. As the dynamics are deterministic, a set of trajectories spanning $\boldsymbol{\Gamma}_{0}$ and $\boldsymbol{\Gamma}_{t}$ (as well as the corresponding set of anti-trajectories) is completely specified by the duration of the trajectories, $t$, and a set of phase-space points at arbitrary time $s, 0 \leqslant s \leqslant t, \delta V\left(\boldsymbol{\Gamma}_{s}\right)$.

A measure of reversibility, $\Omega_{t}$ is defined by the ratio of the probabilities of observing sets of trajectories and their time-reverse or anti-trajectories. The probabilities of the trajectory/antitrajectory can be described by the probabilities of the volume elements at any arbitrary time $s$ along the system's trajectory:

$$
\Omega_{t}(\boldsymbol{\Gamma})=\ln \left[\frac{P\left(\delta V\left(\boldsymbol{\Gamma}_{s}\right)\right)}{P\left(\delta V\left(\boldsymbol{\Gamma}_{s}^{*}\right)\right)}\right],
$$

where we have $\boldsymbol{\Gamma}$ as an argument to the dissipation function, $\Omega_{t}(\boldsymbol{\Gamma})$, to signal that the definition is cast for deterministic dynamics. Equilibrium statistical mechanics provides probability distributions which are simple explicit functions of the phase space, $\boldsymbol{\Gamma}$. In general, it is not possible to cast closed-form expressions of distributions of non-equilibrium states in phase space [6]. However, if we specify that all trajectories are initiated under equilibrium conditions, then the phase-space probability distributions are known initially, $s=0$. The dissipation function is thus written for deterministic systems as $\Omega_{t}(\boldsymbol{\Gamma})=\ln \left[P\left(\delta V\left(\boldsymbol{\Gamma}_{0}\right)\right) / P\left(\delta V\left(\boldsymbol{\Gamma}_{0}^{*}\right)\right)\right]$. As the deterministic definition of $\Omega_{t}$ requires that the relative probabilities of trajectories be made under initial, equilibrium conditions, it is not possible to construct exact expressions for $\Omega_{t}(\boldsymbol{\Gamma})$ for trajectory segments of duration $t$ that are wholly at a non-equilibrium steady state. However, as the dissipation function is extensive, an approximate steady-state dissipation function can be constructed in the following way. We can cast $\Omega_{t}$ in terms of its instantaneous rate of change, $\Omega(s)$ at time $s$, accumulated from an initial equilibrium state at time $s=0$ to some arbitrary time, $t$ :

$$
\Omega_{t}=\int_{0}^{\tau} \mathrm{d} s \Omega(s)+\int_{\tau}^{t} \mathrm{~d} s \Omega(s)
$$

Here we have introduced $\tau$ as an arbitrary 'cut-off' time that is sufficiently large that the system can be regarded as being in steady state for $s>\tau$, so that $\Omega_{t}$ is cast as a sum of transient and steady-state contributions. The steady-state contribution is identified with the steady-state dissipation function, $\Omega_{t}^{\text {ss }}$, which we can use to approximate $\Omega_{t}$ with an error of $\operatorname{order} \tau: \Omega_{t} \approx \Omega_{t}^{\mathrm{ss}}+\mathcal{O}(\tau)$.

An expression for $\Omega_{t}(\boldsymbol{\Gamma})$ is derived in Wang et al [5] for the translating, particle-filled optical trap, and is 


$$
\Omega_{t}(\boldsymbol{\Gamma})=\frac{1}{k_{\mathrm{B}} T} \int_{0}^{t} \mathrm{~d} s\left(\mathbf{f}_{\mathrm{opt}} \cdot \mathbf{v}_{\mathrm{opt}}\right)
$$

Here the dissipation function is the work required to translate the trap. For quasi-static paths where $\mathbf{v}_{\text {opt }} \rightarrow 0$, the dissipation function vanishes, $\Omega_{t} \rightarrow 0$, indicative of a perfectly reversible path. When the trap velocity, $\mathbf{v}_{\text {opt }}$, is finite and the dissipation function $\Omega_{t}>0$ is positive, work is required to translate the trap; in contrast, in those trajectories where $\Omega_{t}<0$, energy is extracted from the surroundings to do work, in contradiction to the average behaviour $\left\langle\Omega_{t}\right\rangle>0$, predicted by the Second Law. Equation (7) is exact when the time integration starts under equilibrium conditions; however, this dissipation function is approximate and equivalent to $\Omega_{t}^{\text {ss }}$ when the time integration starts under non-equilibrium steady-state conditions.

\subsection{Stochastic derivation of the dissipation function for steady-state trajectories}

For some systems whose dynamics can be described using stochastics, it is possible to construct probability distributions of trajectories that are wholly in a non-equilibrium steady-state. The motion of a system under stochastic dynamics is no longer described by the set of coordinates and momenta of all constituent molecules, but is reduced to coordinates, as for example, $\mathbf{r}(t)=\mathbf{r}_{t}$, the position of the colloidal particle trapped in the translating optical trap. Unlike Newtonian dynamics, the stochastic equations of motion cannot be used to construct conjugate pairs of trajectories through time reversal, as the stochastic force is Markovian. Moreover, as the particle position is not unique to any given trajectory, there exist infinitely many trajectories that originate at $\mathbf{r}_{0}$ and a subset of these arrive at a given destination $\mathbf{r}_{t}$ at time $t$. Let $\left\{\mathbf{r}_{0}, \mathbf{r}_{t}\right\}$ represent those stochastic trajectories that evolve from $\mathbf{r}_{0}$ to $\mathbf{r}_{t}$, and let $\left\{\mathbf{r}_{t}, \mathbf{r}_{0}\right\}$ represent a conjugate set of 'backward' trajectories evolving from $\mathbf{r}_{t}$ to $\mathbf{r}_{0}$. Letting $P\left(\mathbf{r}_{0}, \mathbf{r}_{t}\right)$ and $P\left(\mathbf{r}_{t}, \mathbf{r}_{0}\right)$ represent the normalized probability distribution of a set of forward trajectory and respective backward trajectories, then by analogy with equation (5), Reid et al [7] expressed the stochastically determined dissipation function as

$$
\Omega_{t}(\mathbf{r})=\ln \left[\frac{P\left(\mathbf{r}_{0}, \mathbf{r}_{t}\right)}{P\left(\mathbf{r}_{t}, \mathbf{r}_{0}\right)}\right] .
$$

As above, we incorporate $\mathbf{r}$ as an argument to the dissipation function, $\Omega_{t}(\mathbf{r})$, to signal that definition is cast for stochastic dynamics. For a particle in a translating, harmonic potential, it is possible to construct analytic probability distributions $P\left(\mathbf{r}_{0}, \mathbf{r}_{t}\right)$ and $P\left(\mathbf{r}_{t}, \mathbf{r}_{0}\right)$ for steadystate, non-equilibrium particle trajectories that are wholly in a non-equilibrium steady state. In this way, the dissipation function, expressed for a steady-state trajectory of duration $t$, is exactly [5]

$$
\Omega_{t}(\mathbf{r})=\frac{k \mathbf{v}_{\mathrm{opt}} t}{k_{\mathrm{B}} T} \frac{\left(\mathbf{r}_{t}-\mathbf{r}_{0}\right)}{(1-\exp (-t / \tau))},
$$

where $\tau \equiv \xi / k$ is the characteristic timescale of particle motion and $\xi$ is the friction coefficient of the particle.

\section{The experiment}

The equipment used to generate the particle trajectories is similar to that used in the original drag experiment of Wang et al [4]. The apparatus is based upon a Nikon DIAPHOT 300 inverted microscope equipped with a $100 \times(\mathrm{NA}=1.3)$ oil-immersion objective lens and a $1 \mathrm{~W}$ infrared laser $(\lambda=980 \mathrm{~nm})$ for trapping micron-sized particles in the focal plane. An image of a trapped particle is projected onto a quadrant photodiode sensor which can 
detect displacements in particle position with resolution $15 \mathrm{~nm}$. Laser power, objective focus, and servo-motor controlled motion of the microscope stage are controlled through computer interfaces developed by Cell Robotics Inc., USA. Fine translation of the microscope stage is achieved by feeding the voltage signal from an arbitrary function generator (TGA1242, Thurlby Thander Instruments Ltd, UK) to stage-mounted piezocrystals.

Approximately 50 polystyrene particles $(6.3 \mu \mathrm{m}$ in diameter $)$ were added locally into a stage-mounted, glass-bottomed cell, containing a $3.0 \mathrm{ml}$ solution of $10 \mathrm{mM}$ Tris- $\mathrm{HCl}+1 \mathrm{mM}$ EDTA, maintained at a $\mathrm{pH}$ of 7.5. One particle was optically trapped, isolated from the other particles, and used to calibrate the quadrant photodiode detector and optical trap strength. The optical trapping constant, $k$, was determined by sampling the particle's position in a stationary trap for $120 \mathrm{~s}$ at $200 \mathrm{~Hz}$ and applying the equipartition theorem: $k=k_{\mathrm{B}} T /\left\langle r^{2}\right\rangle$, where $r^{2}$ is the square of the displacement of the particle from the focal point, in the focal plane. Particle trajectories, i.e., particle position over time, $\mathbf{r}_{t}$, were then recorded as the stage was translated. A single long trajectory was generated by continuously translating the microscope stage in a circular path. This was achieved by feeding synchronized sine and cosine voltage waves to two perpendicular piezocrystals attached to the microscope stage. The radius of the circular motion was $7.3 \mu \mathrm{m}$ and the frequency of the circular motion was $4 \mathrm{mHz}$. At this low velocity, corresponding to a tangential trap velocity of $\left|-\mathbf{v}_{\text {opt }}\right|=0.18 \mu \mathrm{m} \mathrm{s}^{-1}$, the stage motion can be treated simply as a long linear translation. The trapping constant was determined to be $k=0.12 \mathrm{pN} \mu \mathrm{m}^{-1}$ and the relaxation time of the stationary system was $\tau=0.48 \mathrm{~s}$. This single long trajectory is advantageous for studying steady-state trajectories as it maximizes the amount of steady-state data; only the first few seconds of the initial, transient trajectory are discarded from the analysis. The long trajectory was evenly divided into $75 \mathrm{~s}$ long, nonoverlapping time intervals, then each interval (670 in number) was treated as an independent steady-state trajectory from which we constructed the steady-state dissipation functions using either equation (7), or equation (9).

Figures 1(a) and (b) show the relative frequencies of recorded trajectories with $\Omega_{t}^{\mathrm{ss}}(\boldsymbol{\Gamma})=$ $A \pm \mathrm{d} A$ and $\Omega_{t}^{\mathrm{ss}}(\boldsymbol{\Gamma})=-A \mp \mathrm{d} A$ versus $A$. The time over which the dissipation function is accumulated is $t=0.25$ and $2.5 \mathrm{~s}$ using histogram bins of size $\mathrm{d} A=0.015 / 2$ and $0.15 / 2$ respectively. Referring specifically to figure 1(a), it is clear that the FT is not obeyed-the observed data points do not coincide with the FT prediction. In contrast, the experimental results agree very well with the FT prediction at $t=2.5 \mathrm{~s}$, figure $1(\mathrm{~b})$, and at longer times up to $t=20 \mathrm{~s}$ (not shown), indicating that at long times the FT is obeyed. The scatter at the ends of the distribution can be attributed to finite sampling. Using these figures we have shown that the SSFT is obeyed: the data only match the FT in the long time limit.

Figures 1(c) and (d) show the relative frequencies of recorded trajectories with $\Omega_{t}(\mathbf{r})=$ $A \pm \mathrm{d} A$ and $\Omega_{t}(\mathbf{r})=-A \mp \mathrm{d} A$ versus $A$, where the times over which the dissipation function is accumulated are $t=0.25$ and $2.5 \mathrm{~s}$. From (c) and (d), it is clear that the FT is obeyed at all times using the Langevin-derived dissipation function. Earlier plots at $t=0.05 \mathrm{~s}$ and shorter (not shown) also indicate that the exact, Langevin-derived dissipation function obeys the FT at all times. As the FT can be shown directly using $\Omega_{t}(\mathbf{r})$ we no longer need the long time limit required for the SSFT, and the operative theorem is the FT. The long time limit required for the SSFT clearly arises from the approximations made when deriving the deterministic dissipation function, $\Omega_{t}^{\mathrm{ss}}(\boldsymbol{\Gamma})$.

\section{Conclusions}

In this paper we have directly demonstrated the SSFT, rather than the integrated form of the SSFT previously reported [5]. The data show that the FT is the applicable theorem when the 

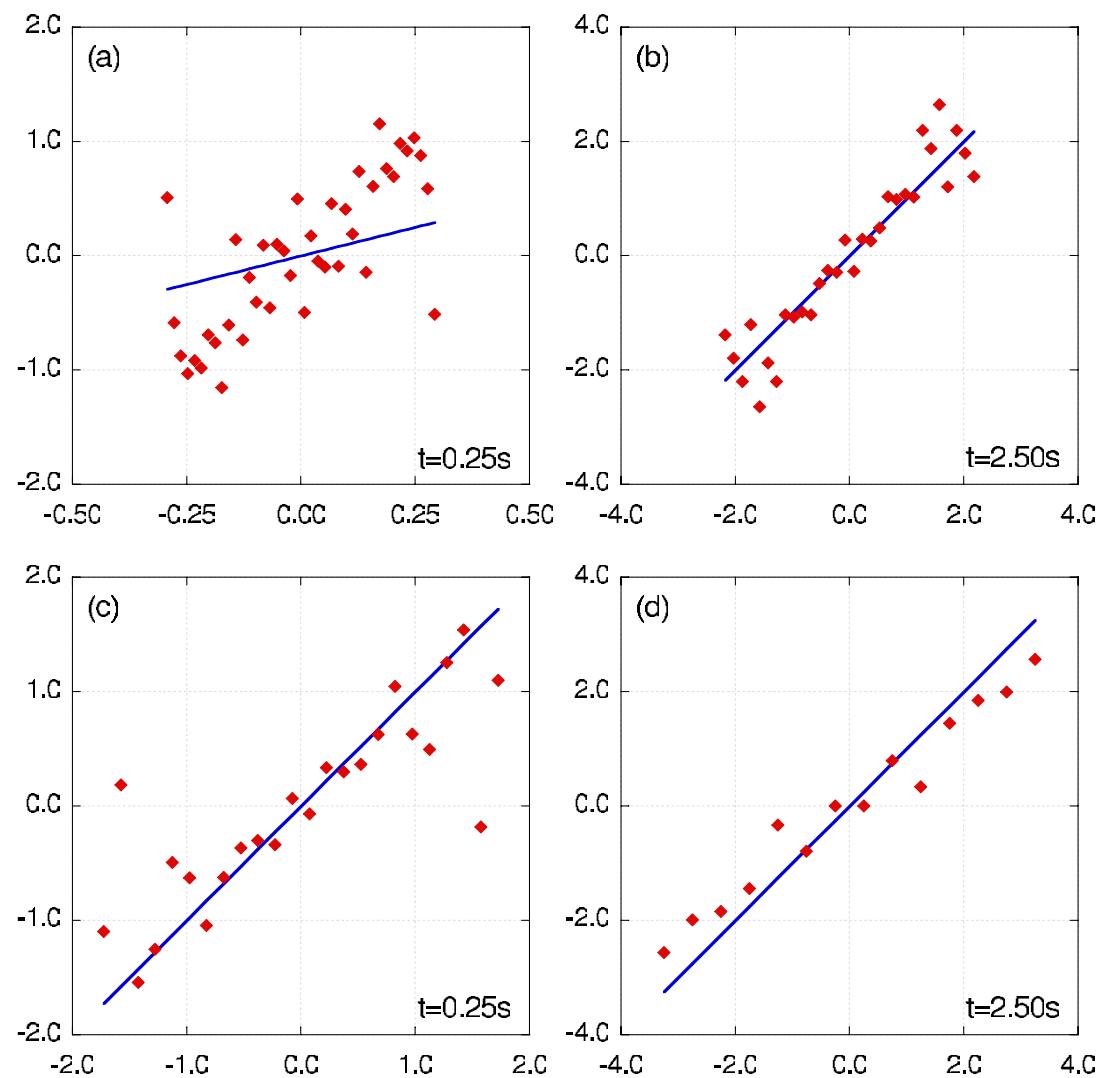

Figure 1. In each figure $\ln \left[P\left(\Omega_{t}=A\right) / P\left(\Omega_{t}=-A\right)\right]$ is plotted against $\Omega_{t}$. The theoretical FT prediction is shown as a continuous line. (a) The FT using $\Omega_{t} \equiv \Omega_{t}^{\mathrm{ss}}(\boldsymbol{\Gamma})$ is plotted at $t=0.25 \mathrm{~s}$ using a histogram bin width of 0.015 . (b) The FT using $\Omega_{t} \equiv \Omega_{t}^{\mathrm{ss}}(\boldsymbol{\Gamma})$ is plotted at $t=2.5 \mathrm{~s}$ using a histogram bin width of 0.15 . (c) The FT using $\Omega_{t} \equiv \Omega_{t}(\mathbf{r})$ is plotted at $t=0.25 \mathrm{~s}$ using a histogram bin width of 0.15 . (d) The FT using $\Omega_{t} \equiv \Omega_{t}(\mathbf{r})$ is plotted at $t=2.5 \mathrm{~s}$ using a histogram bin width of 0.50 .

(This figure is in colour only in the electronic version)

dissipation function is derived exactly. But when approximations are made to the dissipation function, the FT only holds in the long time limit, thus illustrating the previously published form of the SSFT. Our data also show that the long time limit in the SSFT is a direct result of approximations made when casting expressions for the dissipation function.

\section{References}

[1] Evans D J, Cohen E G D and Morriss G P 1993 Phys. Rev. Lett. 712401

[2] Evans D J and Searles D J 1994 Phys. Rev. E 501645

[3] Searles D J and Evans D J 2004 Aust. J. Chem. 57121119

[4] Wang G M, Sevick E M, Mittag E, Searles D J and Evans D J 2002 Phys. Rev. Lett. 89050601

[5] Wang G M, Reid J C, Carberry D M, Williams D R M, Sevick E M and Evans D J 2005 Phys. Rev. E 71046142

[6] Evans D J and Morris G P 1990 Statistical Mechanics of Nonequilibrium Liquids (San Diego, CA: Academic)

[7] Reid J C, Carberry D M, Wang G M, Sevick E M, Searles D J and Evans D J 2004 Phys. Rev. E 70016111 